\title{
The Effects Of Caffeine On Athletic Performance
}

\author{
Larry W. McDaniel, Dakota State University, USA \\ Kyle McIntire, Dakota State University, USA \\ Carmyn Streitz, Dakota State University, USA \\ Allen Jackson, Chadron State College, USA \\ Laura Gaudet, Chadron State College, USA
}

\begin{abstract}
Athletes who use caffeine before exercising or competition may be upgrading themselves more than they realize. Caffeine is classified as a stimulant and is the most commonly used drug in the world. Caffeine has the same affects that amphetamines and cocaine have, just to a lesser degree. Caffeine crosses the membranes of all the body's tissues. It can exert effects on the central nervous system and the peripheral tissues that result in physiological effects. Studies have shown that caffeine improves performance in a variety of different activities. This stimulant has been shown to be a powerful ergogenic aid that is beneficial in athletic training and performance. Caffeine has been found to increase speed and power, improve the length of training, and assist the athlete in resisting fatigue. Caffeine has been found to stimulate the brain, which contributes to clearer thinking and ability to concentrate more intensely on the task at hand. Studies have shown that up to 25\% of athlete's ages 11-18 years old have used caffeine in an effort to increase their athletic performances. Because of caffeine's effect on the body and its ability to increase an athlete's performance, Olympic Committees have debated on whether caffeine should be tested before the Olympic Games.
\end{abstract}

Keywords: Caffeine; Caffeinated Drinks; Muscle Glycogen; Exercise; Performance Enhancing Drugs

\section{INTRODUCTION}

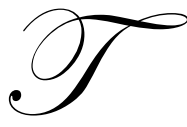

he purpose of this paper is to explore caffeine's affect on athletic performance. An additional focus will be to search for information related to the benefits of caffeine that may improve athletic performance.

\section{THE BROAD USE OF CAFFEINE}

"Caffeine is classified as a stimulant and is performance enhancing" (Jenkinson \& Harbert, 2008, pg. 3). Caffeine is the most commonly used drug. In general, society would not approve of an athlete using a steroid drug or a stimulant, but no one reacts negatively to athletes drinking coffee, tea, energy drinks, or soft drinks. Caffeine is appearing in a number of other products, including energy drinks, bars, sports gel, alcoholic beverages, and diet aids. Figure 1 shows a comparison of caffeinated drinks, many of which are used by athletes before a competition.

The American Alliance for Health stated that there are three ways that caffeine may provide ergogenic effects. "First, the metabolic theory suggests that caffeine provides improved endurance due to an increased utilization of fat as fuel and a sparing effect on carbohydrate utilization. Secondly, caffeine may increase the calcium content of the skeletal muscle and enhance the strength of muscle contraction. Lastly, caffeine has a direct effect on the central nervous system as a stimulant, which can help with fatigue, increased alertness, and increased muscle recruitment" (Powers M, 2004, pg. 4). 
For years, many athletes have used caffeine prior to competitions, but it wasn't until recently that caffeine has been discovered to aid an athlete's performance. "Results of studies reported over the last five years strongly indicate that caffeine effectively increases athletic performances in endurance events" (Sinclair \& Geiger, 2000, pg. 2). Athletes ranging from long distance runners to those participating in strength and power competitions benefit from caffeine consumption. "Persons were able to complete a cycling time trial significantly faster after caffeine ingestion and 2,000-meter rowing time was reduced by 1.2 percent after caffeine ingestion" (Jenkinson \& Harbert, 2008, pg. 3). Caffeine is a common substance found in the diets of many athletes; therefore, it is important to study the effects on the athletes' body. "Caffeine operates using the same mechanisms that amphetamines and cocaine use to stimulate the brain; however, the effects are milder because it crosses the membranes of all tissues in the body" (Powers M, 2004, pg. 4). Caffeine is a powerful ergogenic aid that may be beneficial in training and athletic performance. "It can exert its effects on both the central nervous system and the peripheral tissues, resulting in a number of physiological effects that might improve performance" (Powers M, 2004, pg. 4). Caffeine has been shown to increase speed and power. It also allows athletes to train longer. Caffeine stimulates the brain, which contributes to clearer thinking and greater concentration. Studies have shown that caffeine doesn't directly improve maximal oxygen capacity, but assists in the process of resisting fatigue. "Although the effectiveness of caffeine as a means of masking fatigue has been explored since the early 1900s, the use of this ergogenic aid became popular following widely publicized research indicating improved endurance performance" (Applegate \& Grivetti, 1997, pg. 6). Like all drugs, caffeine use has side effects. There is no evidence that states that caffeine leads to dehydration, ion imbalance, or any other adverse effects.

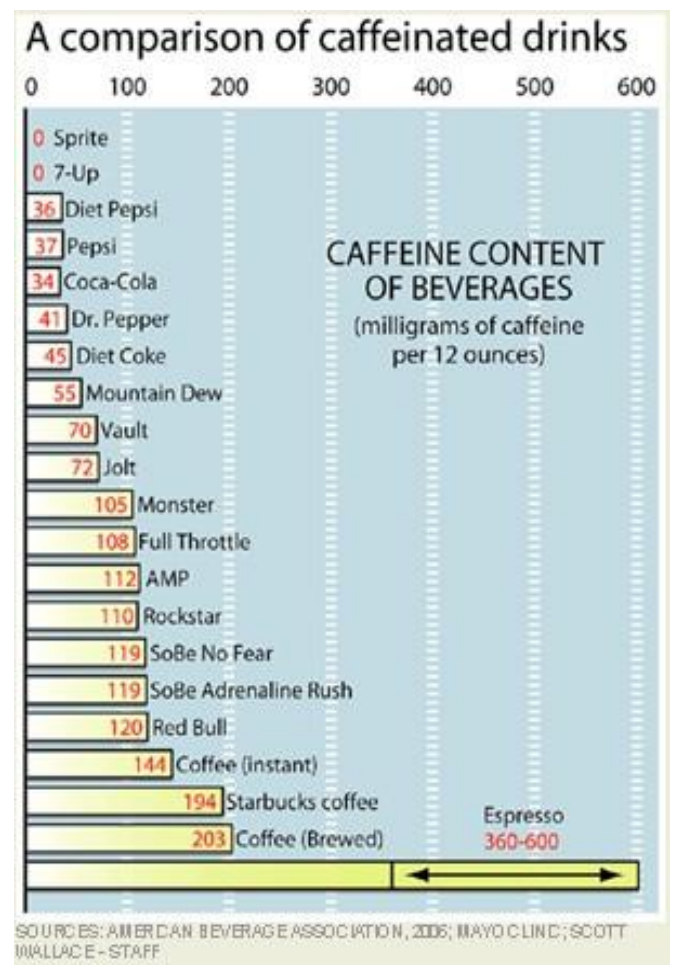

\section{CAFFEINE USE}

"Caffeine is the most widely ingested psychoactive drug in the world" (Sinclair \& Geiger, 2000, pg. 1). Caffeine can lead to dependence, tolerance, drug craving, and withdrawal symptoms when it is used chronically; however, it is still a legal substance used in sports. This stimulant is known to allow athletes to train harder and longer. "Drug use among athletes for the purpose of achieving athletic benefit starts at a very young age. Of 11-18 year-old students surveyed, $35 \%$ believed caffeine could enhance performance and $25 \%$ used caffeine in an effort to 
increase athletic performance" (Sinclair \& Geiger, 2000, pg. 2). The stimulant caffeine affects the brain which contributes to clearer thinking and greater concentration. An article published in the American Alliance for Health, Physical Education, and Recreation (2004) stated, "There appear to be three primary mechanisms by which caffeine might provide an ergogenic effect. First, the metabolic theory suggests that caffeine provides improved endurance due to an increased utilization of fat as a fuel and a sparing effect on carbohydrate utilization. Secondly, caffeine may increase the calcium content of skeletal muscle and enhance the strength of muscle contraction. Finally, caffeine has direct effects on the central nervous system as a stimulant which may alter the perception of fatigue, increase alertness, and increase muscle recruitment. An endurance athlete may be more likely to consider using the first mechanism, while the latter two would more likely be used by athletes participating in strength and power competitions and events requiring arousal and alertness" (Powers M, 2004, pg. 4).

Caffeine may be absorbed in several different ways and can be administered orally, through injections administered either subcutaneously or intramuscularly, or by suppositories. "The actions of caffeine throughout the body correlate positively with caffeine levels and the levels are governed by caffeine absorption, metabolism and excretion. Caffeine is absorbed efficiently through the gastrointestinal tract after oral administration with about $100 \%$ bioavailability" (Sinclair \& Geiger, 2000, pg. 2).

\section{SPEED/POWER IN LONG TERM EXERCISE}

There have been few studies conducted to evaluate the effects that caffeine has on speed or endurance events. Early studies found improvements in activities such as repeated jumping, bench stepping, cycling, and treadmill tests. Researchers have studied elite skiers on a 20-23 km course at both high and low altitude. The ingestion of caffeine resulted in faster performance times at the halfway mark and the finish line. The total time was about 55-67 minutes, while caffeine resulted in times of 33 and 101 seconds faster for low and high altitudes. The caffeinated athletes' generated $7.3 \%$ greater total power output. Skilled cyclists were told to perform, as quickly as possible, a set amount of work that was estimated to take about an hour. After exercising to exhaustion, seven endurance cyclists were given either a straight carbohydrate drink or one laced with the equivalent of six cups of coffee. "While it's been established that carbohydrates and caffeine improve a variety of athletic performances, this is the first study that has revealed that combining caffeine with carbohydrates after you've exercised can actually help your muscles refuel more rapidly" (Caffeine Aids Athlete Recovery, 2008, pg. 1). When the solution contained caffeine, the power output improvement was greater.

\section{POWER IN SHORT TERM, INTENSE EXERCISE}

The ability to perform at high intensity has been examined in several studies. In a similar study, participants simulated a $1500 \mathrm{~m}$ run, and coffee ingestion produced a 4.2 second improvement in running speed. Dr. Collomp studied swimmers who swam $100 \mathrm{~m}$ freestyle. Caffeine ingestion significantly improved the mean time of highly trained simmers by about 1 second, while untrained athletes showed no improvement. When activities of shorter duration were examined, the results are more inconsistent, probably because the potential improvement is small and difficult to measure. These areas are not well studied, but it appears exercise lasting at least 60 seconds proves caffeine as an ergogenic aid and there are no studies showing negative effects.

\section{MUSCLE GLYCOGEN}

Studies have demonstrated that caffeine results in glycogen sparing. Professor John Hawley, Head of RMIT's Exercise Metabolism Group, found that athletes who had caffeine with their meal after exercise had $66 \%$ more glycogen in their muscles 4 hours later (Caffeine Aids Athlete Recovery, 2008). Glycogen is the body's preferred fuel for muscles when exercising. Hawley Stated, "If you have $66 \%$ more fuel for the next day's training or competition, there's no question you'll be able to go further and faster (Caffeine Aids Athlete Recovery, 2008, pg. 1)". There are many experiments lasting less than 30 minutes in which caffeine has been shown to be beneficial when glycogen does not appear to be limiting. 


\section{CONCLUSION}

Caffeine is a complex substance that is found in many organic compounds and is consumed by humans in coffee, tea, and chocolate. Caffeine is the most commonly used drug in the world. Food industries are adding caffeine to a wide variety of foods and drinks. Caffeine is found in a number of 'natural health products' and in many over-the-counter drugs. The affect caffeine has on the body ranges from various adenosine receptors in several types of body tissues. Caffeine is ergogenic in most, if not all, aerobic exercises. Studies have shown that as an ergogenic aid, caffeine enhances endurance-type exercises, such as running, swimming, cycling, and tennis. Caffeine also provides benefits in anaerobic activities, such as resistance training. Olympic committees are debating whether or not caffeine should be tested prior to the Olympic Games because of its ergogenic effects. "Glucose recovery slows drastically after 3-4 hours, so recovery rates after 4 hours are excellent proxies for glycogen storage 24 hours after exercise. If you have 66\% more fuel for the next day's training or competition, there's no question you'll be able to go further and faster" (Caffeine Aids Athlete Recovery, 2008, pg. 1). So far, there has been little evidence demonstrating that the administration of caffeine substances prior to or after exercise produces a negative effect. The mechanisms involved in actions of caffeinated products are varied, complex, and extend beyond traditional explanations of sparing of muscle glycogen to probably involving fundamental aspects of muscle contractility.

\section{AUTHOR INFORMATION}

Larry W. McDaniel Ed.D. is an Associate Professor of Exercise Science at Dakota State University Madison, SD. USA. Dr. McDaniel was a First Team All-American football player (USA Football), a Hall of Fame Athlete, and Hall of Fame Wrestling Coach.

Allen Jackson, M. Ed. is an Assistant Professor of Physical Education and Health at Chadron State College in Chadron, Nebraska (USA) who is well known for his presentations and publications at international conferences focusing on Leadership, Curriculum, and Health.

Laura Gaudet, Ph.D. is a Professor and Chair of the Department of Counseling, Psychology and Social Work at Chadron State College, Chadron NE. Dr. Gaudet is well known for her publications and presentations at international conferences focusing on various topics in the field of psychology.

Kyle McIntyre is an outstanding athlete and student of Exercise Science at Dakota State University.

Carmyn Streitz is an outstanding athlete and student of Exercise Science at Dakota State University.

\section{REFERENCES}

1. $\quad$ Antonio J. (2004) "Caffeine: The Forgotten Ergogenic Aid." Strength and Conditioning Journal 26.6 (2004): 50-51. Research Library. ProQuest. Karl E. Mundt Library, Madison, SD. 22 Jan. $2009<$ http://www.proquest.com/>

2. Applegate E. \& Grivetti L. (1997). Search for the competitive edge: A History of dietary fads and supplements. The Journal of Nutrition: 1996 ASNS Symposium Proceedings, 127(5S), 869S-873S. Retrieved October 30, 2009, from Research Library. (Document ID: 11802051).

3. Beaven C., Martyn, et al. (2008) "Dose Effect of Caffeine on Testosterone and Cortisol Responses to Resistance Exercise." International Journal of Sport Nutrition \& Exercise Metabolism 18.2 (Apr. 2008): 131-141. EBSCO MegaFILE. EBSCO. [Library name], [City], [State abbreviation]. 29 Jan. 2009

4. Caffeine Aids Athlete Recovery. "Australasian Science 1 Sep. 2008: 6. ProQuest Education Journals. ProQuest. Karl E. Mundt Library, Madison, SD. 29 Jan. 2009 <http://www.proquest.com/>

5. Clark N. (2005) "Caffeine and Performance." Palaestra 1 Oct. 2005: 46. Research Library. ProQuest. Karl E. Mundt Library, Madison, SD. 22 Jan. 2009 <http://www.proquest.com/>

6. Graham T., (2001) "Caffeine and Exercise: Metabolism, Endurance and Performance." Sports Medicine 31.11 (Aug. 2001): 785-807. EBSCO MegaFILE. EBSCO. [Library name], [City], [State abbreviation]. 29

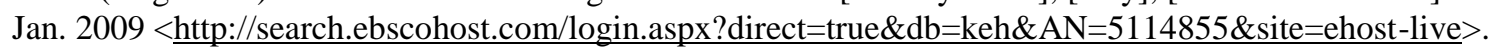


7. Jenkinson, D., \& Harbert, A. (2008). Supplements and Sports. American Family Physician, 78(9), 103946. Retrieved October 30, 2009, from Research Library. (Document ID: 1584143711).

8. McNaughton L., Lovell R., Siegler J., Midgley A., Sandstrom, M., \& Bentley D.. (2008). The effects of caffeine ingestion on time trial cycling performance. Journal of Sports Medicine and Physical Fitness, 48(3), 320-5. Retrieved October 30, 2009, from ProQuest Education Journals. (Document ID: 1592003301$)$.

9. Powers M. "Safety, Efficacy, and Legal Issues Related to DIETARY SUPPLEMENTS. " Strategies 18.1 (2004): 30-34. ProQuest Education Journals. ProQuest. Karl E. Mundt Library, Madison, SD. 22 Jan. 2009 <http://www.proquest.com/>

10. Sinclair C., \& Geiger J. (2000). Caffeine use in sports: A pharmacological review. Journal of Sports Medicine and Physical Fitness, 40(1), 71-9. Retrieved October 30, 2009, from ProQuest Education Journals. (Document ID: 70976173).

\section{PICTURE REFERENCES}

1. A Comparison of Caffeinated Drinks [Photograph]. (2006). Retrieved December 4,2009, From: http://biolife.files.wordpress.com/2008/04/caffeinated-drink-comparison.jpg

2. Effects of Caffeine on Endurance Performance [Table]. (1998). Retrieved December 4, 2009, From: http://proquest.umi.com/pqdweb/nosession/nosession?des=R0tCGn7IHU9XybtfrjvJAlSLqAskewda30rkG MScarEJtVMqmBWKTpdewP9ghsokE1jZutfdVNEs+P+BHrl7ztSTpIzFCayN9d4M/88U4A8YtZC11q25 UeUgNXO9nm+9gQdlr3aAZvjkgrXjFw0rG59vKVnLz37kFh1bTEIODmunTbdslt7iKHHg3qBdOruqJNN $\mathrm{Vn3cNwCa+KsXu/W+m2BoQ/rm9ayBQQ4Em2Fjs4Us= \& mac=6fa3e46f41dc1fb3a6be7ed95e3c4645}$ 


\section{NOTES}

